TORNAY, Serge. - Les Fusils Jaunes. Générations et politique en pays nyangatom (Éthiopie). ParisNanterre, Société d'ethnologie, 2001, 303 p.

\title{
Claude Rivière
}

\section{(2) OpenEdition}

Édition électronique

URL : http://journals.openedition.org/etudesafricaines/5495

DOI : 10.4000/etudesafricaines.5495

ISSN : $1777-5353$

Éditeur

Éditions de l'EHESS

\section{Édition imprimée}

Date de publication : 25 juin 2005

ISBN : 978-2-7132-2048-7

ISSN : 0008-0055

\section{Référence électronique}

Claude Rivière, «TORNAY, Serge. - Les Fusils Jaunes. Générations et politique en pays nyangatom

(Éthiopie). Paris-Nanterre, Société d'ethnologie, 2001, 303 p. », Cahiers d'études africaines [En ligne],

178 | 2005, mis en ligne le 30 juin 2005, consulté le 24 septembre 2020. URL : http://

journals.openedition.org/etudesafricaines/5495; DOI : https://doi.org/10.4000/etudesafricaines.5495

Ce document a été généré automatiquement le 24 septembre 2020.

(C) Cahiers d'Études africaines 


\title{
TORNAY, Serge. - Les Fusils Jaunes.
} Générations et politique en pays nyangatom (Éthiopie). Paris-Nanterre, Société d'ethnologie, 2001, 303 p.

\author{
Claude Rivière
}

1 Cet ouvrage représente la synthèse des recherches ethnographiques que l'auteur a conduites depuis 1970 dans la basse vallée de l'Omo en Éthiopie. En 1989, il soutenait, sous la direction du regretté Éric de Dampierre, une thèse d'État qui mettait en évidence le système social des Nyangatom comme un système générationnel: une première dans les recherches africanistes françaises. Les Fusils Jaunes ne sont pas le résumé de cette thèse, mais un essai mûri après plusieurs retours sur le terrain au cours des années 1990.

2 Les Nyangatom, groupe minoritaire du cercle karimojong et dont Tornay demeure à ce jour le seul ethnographe, vivent à l'extrême sud-ouest de l'Éthiopie, aux confins du Soudan et du Kenya. Cette zone frontière fut conquise par l'empereur Ménélik en 1899 mais l'occupation amhara demeura purement nominative et la région n'a pas été colonisée. En cinq chapitres sont traités les problèmes de production dans l'écosystème, l'historique du groupe, le système de parenté et d'alliance, les parades initiatiques et les festivités sacrificielles de ceux qui préfèrent s'appeler par leur nom de guerre Nyang-atom, c'est-à-dire "fusils jaunes » que par leur ancien sobriquet de " mangeurs d'éléphants »: Nyam-etom.

En introduction, l'auteur présente ce qu'ont d'original les manières de classer les individus en catégories selon les éléments de leur statut et de se reconnaître comme Éléphant ou Autruche bien qu'il n'existe point de patronyme mais seulement un nom de naissance et une appartenance territoriale (par exemple celle des Cigognes, des Flamants, des Ibis) garantissant les droits d'accès aux ressources. Sans système politique centralisé ni rapport de dominant-dominé avec leurs voisins, mais sensibles au pouvoir charismatique, ils sont à division non pas clanique ou lignagère mais 
générationnelle. La génération est subdivisée en classes d'âge et celles-ci en groupes locaux.

4 Dans le système générationnel, le lien Père du pays-Fils du pays, qui représente la structure proprement politique de la société, est l'expression directe d'une logique d'identification des générations alternes et d'opposition des générations successives. Sont donc, en un temps donné, Pères du pays, les hommes de la génération en titre, mais également leurs petits-fils et, s'il en subsiste, leurs grands-pères. Les Fils du pays sont les enfants des Pères en titres, mais également leurs grands-pères et leurs petitsfils. Ainsi donc l'ensemble de la population est-elle répartie en deux classes et deux statuts seulement, ceux de Pères et de Fils. L'originalité du système générationnel - que Tornay défend à la suite d'une étude pionnière de Paul Spencer sur les Jie, eux aussi membres du cercle karimojong - consiste dans la périodicité longue de la succession des générations. Alors que dans les familles, les générations émergent, pour ainsi dire mécaniquement tous les vingt-cinq ans environ, à l'échelle de la société globale, c'est un phénomène statistique qui entre en jeu : l'intervalle générationnel est d'un peu plus de cinquante ans, ce qui n'est pas sans évoquer, pour l'africaniste, le rythme long du sigui des Dogon. Tornay, suivant la géniale impulsion de Spencer, a recueilli les données démographiques seules capables de démontrer la réalité du système générationnel. Pour l'Afrique orientale, il a même fait école, si l'on peut dire, puisque Anne-Maris Peatrik, auteure d'une monographie remarquée sur les Meru du Kenya, fut à Nanterre, élève de Tornay. Peuples de langue bantu, les Meru, comme les Kikuyu, ont également des systèmes générationnels, lesquels demeurèrent pratiquement incompris jusqu'à cette dernière décennie. Chez les Nyangatom, l'essence politico-religieuse du système est que les fils doivent nourrir leurs pères, quelle que soit la génération à laquelle ils appartiennent. Mais s'agissant du statut, c'est une même moitié de la société qui demeure "à la paternité » du pays, donc à la position dominante, pour une durée d'environ cinquante ans, puisque telle est l'inertie démographique du système. Cette situation ne va pas sans accumuler des frustrations et c'est, pour autant que l'ethno-histoire s'en souvienne, au terme d'une période de quasi-rébellion que les Pères en titre acceptent de passer la main à leurs fils, provoquant ainsi l'alternance politique dans la société. Évidemment, cette alternance ne relève pas d'un processus à l'anglo-saxonne, mais est bien l'expression du principe de séniorité qui découle du fait biologique et social de l'engendrement. Parallèlement aux garçons de la même génération, les filles ont leurs propres groupes d'âge qu'elles quittent en se mariant.

5 Est ensuite présentée au lecteur la culture matérielle de ces cinq mille personnes environ qui récoltent sorgho, maïs, haricots, ramassent le miel, sèchent des poissons qu'ils pêchent, le temps de vivre dans quelque éphémère camp pastoral près de voisins mobiles et hostiles le plus souvent tels les Turkana ou les Dassanetch leur disputant herbages et bétail. Dans l'année, mesurée par les lunaisons et démarrant avec les premières pluies, alternent inondations avec concentration humaine et sécheresse entraînant la dispersion des groupes. Pour richesse, dans cette culture du dénuement mais cependant du savoir-faire, l'homme a son troupeau, ses femmes et ses enfants, mais aussi ses ornements corporels, son appuie-tête et ses armes (p. 68).

6 Le chapitre suivant, "Ethno-génèses ", s'ouvre par une mise en place historique et géographique tout à fait remarquable de l'ensemble des populations du Haut Nil, des divisions et migrations de groupes apparentés dans les deux derniers siècles, inspirée 
par Lamphear et Pazzaglia notamment. On y voit l'originalité absolue du système générationnel des Karimonjong par rapport au système des classes d'âge à initiation périodique de cohortes de contemporains, attesté chez les Kalenjin, Samburu, Massai... La mémoire des peuples ne se nourrit pas là de célébrations du passé mais des expéditions guerrières, des offrandes filiales et des bénédictions du présent. À la riche documentation d'histoire orale constituée d'entretiens variés et de leurs commentaires s'adjoignent le mythe d'une femme au sein unique et des évocations d'Italiens venus avant les Amhara qui « eux cherchent la bagarre ", ainsi que des hypothèses prudentes sur la succession des générations et sur les possesseurs du pays.

7 Côté famille et parenté, la patrilinéarité fait que le père contrôle la production économique des fils de ses épouses. En respectant une règle de précédence selon le rang de mariage, chaque épouse gère sa progéniture et ses biens, mais les fils doivent nourrir les pères qui possèdent le troupeau, lequel sert éventuellement à régaler les pairs lors de festins cérémoniels. Comme élément important de la compensation matrimoniale entrent aussi une trentaine de têtes de gros bétail et quelques centaines de chèvres et de moutons, ce qui entraîne une dette à vie ou du moins jusqu'à clôture festive du paiement. À sa mort, l'ancien a sa place dans l'enclos aux zébus sous un monticule de bouses sèches et les deuilleurs se blanchissent le corps de farine de sorgho. Quant aux mauvais morts, on les abandonne aux vautours et aux hyènes. À l'issue du chapitre et de la première partie de l'ouvrage, l'esprit de Tornay éclate en un feu d'artifice de six pages d'argumentaire (pp.183-188) sur le système générationnel que doivent lire tous les africanistes, et tellement compact qu'il me semble impossible à résumer, tout comme d'ailleurs la postface (pp.325-331) reprécisant que la génération ne se définit pas à partir de la filiation mais comme cohorte historique.

8 La même élégance du style, la forte concision des idées me rappellent quelques articles du même auteur sur les systèmes de parenté de l'Est africain redisant ses compétences déjà exprimées dans plusieurs articles de L'Homme et dans un premier chapitre de L'anthropologie, science des sociétés primitives? sous la direction de Maurice Godelier. On ne peut qu'apprécier l'alternance intéressante de réflexions théoriques, de descriptions du vécu, de notations tristes (Les chameaux emportés par la maladie, les dysenteries causées par le ver du sorgho se développant près des mares) ou de réponses rieuses dans le vif de l'entretien («Vous permettez que j'aille abreuver mes aïeuls de l'eau chaude de Tornay ! », dit Kotol, et il sort de la hutte pour se soulager).

9 La seconde partie est plutôt politique et religieuse. L'offrande filiale représente l'acte de reconnaissance de l'autorité. Par l'initiation et le sacrifice sont confirmés les statuts de membre de la société et transmise la souveraineté des Pères aux Fils du pays. Et les affaires étrangères de s'éclairer par des aventures guerrières contre des tribus voisines pour meurtre de femme ou de berger, refus d'accès aux puits, pression migratoire après tribulation climatique, sans parler des razzias de bétail en représailles ou pour manifester la vigueur des jeunes. Histoires de butin, crise d'identité après défaite, malédictions contre les Amhara en plantant la lance de parole, appel des trompes, voilà ce que saisit fort bien l'ethnographe et qu'il restitue dans son journal de route fort précis, où sont notés l'hymne au bœuf-tête-de-bois, l'aspersion avec le goupillon de filasse, les bénédictions par jet de salive, les onctions d'hématite et de beurre sur la poitrine et les cuisses du guerrier.

10 Nombreux paraissent les rites, mais sans référence à des mythes et seulement pour honorer les vivants et les ancêtres. Quant aux sacrifices de bétail, ils n'impliquent pas 
comme destinataires des forces surnaturelles ou une instance divine, mais traduisent un hommage politique, sans décorum théologique, à l'élément sacré de la société en l'absence du roi et de l'État. «Sacrifier aux Pères, c'est s'initier en tant que Fils du pays. Dans cette Afrique sans masque, l'initiation ne fait pas de mystère " (p. 272). Après une critique très fine des interprétations d'Evans-Pritchard concernant le sacrifice nuer, l'auteur distingue : 1) le sacrifice-festin-convivial adressé aux Pères et aux pères des Pères, qui recompose le corps social ;2) l'immolation plus magique que religieuse d'un animal à finalité de divination, d'appel du succès, de fécondité pour les Nyangatom ou de mort des ennemis ; 3) le sacrifice-clé de la transmission générationnelle : l'hommeasapan, " cet individu choisi et désigné va sacrifier en l'égorgeant le bœuf coutumier ; puis il va imposer ses mains sur les Fils du pays pour les transformer en Pères, après quoi son destin annoncé, la perte de la raison, le conduit à la mort " dans la brousse (p. 282). Voilà bien illustrée, dans une société pourtant sans souverain, la thèse de Frazer sur la mise à mort du chef comme victime de substitut pour revitaliser le groupe!

11 L'initiation première dans cette population est offrande et immolation d'un animal castré pour régaler l'assemblée des anciens. L'identité sociale est ainsi engendrée par le sacrifice de type 1. L'assurance du bien-être s'obtient par un rite du type 2. L'initiation de passage de la génération des Fils (généralement cinq classes d'âge) au statut de Pères s'accomplit par un sacrifice de type 3 dont sont décrits les rites de manière minutieuse et vivante. Et l'ouvrage se clôt par des réflexions pertinentes sur le blocage possible du système générationnel.

12 D'un directeur adjoint au Musée de l'homme, naguère professeur à l'Université de Nanterre, on ne peut que s'attendre à une œuvre d'excellence, ce dont témoignent la manière de rendre présents les gens et les choses, les comparaisons opérées entre ethnies voisines; les discussions de textes d'auteurs majeurs ayant abordé tel sujet traité ici, les schématisations de la cosmologie, les classifications par correspondance entre espèces animales, végétales et groupes humains. Même si la gestion politique de la société nyangatom paraît insuffisamment analysée, même si la compréhension du système générationnel ou de types particuliers d'initiation demande un certain effort au lecteur, celui-ci sera récompensé par l'agrément du voyage en des contrées peu familières et par un jet de salive du maitre des lieux ayant valeur de bénédiction. L'apprenti ethnologue y apprendra comment creuser un puits... de science. 